\title{
Force analysis of clogging arches in a silo
}

\author{
R. C. Hidalgo • C. Lozano • I. Zuriguel · \\ A. Garcimartín
}

Received: 15 June 2013

(C) Springer-Verlag Berlin Heidelberg 2013

\begin{abstract}
In this work, we examine a quasi-2D silo that clogs due to the spontaneous formation of stable arches. We validate a numerical scheme comparing the morphology of clogging arches with previous experimental findings. Additionally, we inspect the forces that act on particles, both on those in the bulk of the silo as well as those belonging to the arches formed above the outlet. In the silo, we have found that normal forces are higher close to the wall, in contrast to the central part of the silo, where normal forces are notably lower. Besides, it is revealed that normal forces on particles belonging to the clogging arches are significantly larger than in their surroundings. In a particle of the arch, the magnitude of the force strongly depends on the angle subtended from its center to the contact points with its two neighbors in the arch. Indeed, for angles exceeding $180^{\circ}$, the larger the angle, the lower the normal force and the higher the tangential one. On the contrary, for smaller angles the behavior is reversed, so the normal forces increase with the angle. Finally, we present a comparison of the normal and tangential force distributions for the particles within the arch and in the bulk. All this shows the special nature of the forces developed in clogging arches, which suggests that direct extrapolations of bulk properties should not be taken for granted.
\end{abstract}

Keywords Arch $\cdot$ Bridge $\cdot$ Clogging $\cdot$ Jamming $\cdot$ Silo

\section{Introduction}

Arches (or bridges) are usually found in particulated materials such as granular media, colloids, suspensions or crowds.

R. C. Hidalgo $(\bowtie) \cdot$ C. Lozano · I. Zuriguel · A. Garcimartín Departamento de Física y Matemática Aplicada, Facultad de Ciencias, Universidad de Navarra, 31080 Pamplona, Spain e-mail: raulcruz@unav.es
They can be defined as an assembly of elements which are mutually stabilized under gravity or any other external field [1-4].

Two different approaches have been used to describe the complex structure of arches. On one hand, several groups have explored the nature of arches within the bulk of granular media [1-7] or colloidal samples [7,8]. This course has led to useful analogies between the properties of arches and those of the force chains. Interestingly, it has been found that the nature of two dimensional and three dimensional arches differs. Indeed, in three dimensions two different kind of bridges are found, namely, simple linear arches and complex $3 D$ backbones with loops and/or branches [1-4].

On the other hand, several authors have focused their attention on the arches that arise at a bottleneck. These arches seem to be crucial to understand clogging, which is a major problem when a particulated material is forced to pass through an stranglement. Familiar examples of clogging appear in crowd stampedes $[9,10]$, fluid-driven particle flows [11,12], and granular materials. In this later case, abundant research has been carried out covering $2 D[13,14]$ and $3 D[15,16]$ silos, inclined outpouring $[17,18]$, silos with an obstacle above the outlet [19], anisometric (wedge) hoppers [20], and piles discharged through an orifice at their bottom [21]. In all these systems clogging arches withstand the weight of the particles above them just as architectural arches do. For the case of silos, a link has been sought between the properties of the arches and the clogging probability $[13,14,22,23]$. Although the relationship is not fully understood, the knowledge about the properties of these assemblies has been notably improved.

To et al. [13], To and Lai [14] reported that clogging arches have a semi-circular convex shape, which can be reproduced by a restricted random walk model. Note that a convex shape is, indeed, a necessary condition for the static equilibrium of 


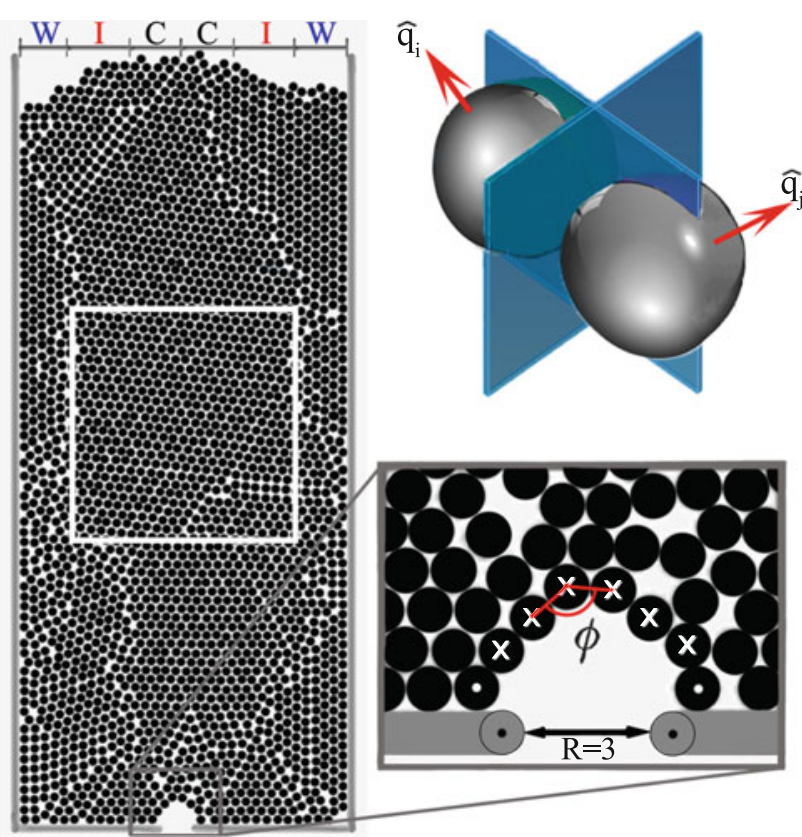

Fig. 1 Sketch of the simulated silo. At the left the silo is represented, with an indication at the top of the different regions in which forces have been analyzed ( $W$, wall; $I$, intermediate; and $C$, central). The big white square marks the zone that has been considered as the bulk region, as justified in the text. At the bottom right, a close up of the outlet neighborhood is displayed with an arch. Particles belonging to the arch (to the base of the arch) are marked with crosses (dots). The angle associated to one of the particles in the arch $(\phi)$ is also drawn. The outlet is formed by two particles whose centers are $4 d$ apart, giving an outlet size of $R=3$. At the top right, a sketch of the interaction among two particles is shown

frictionless particles. If friction is the case, Garcimartín et al. [24] showed that the convex condition is not always fulfilled locally. They measured the angle $\phi$ formed by each particle and its two neighbors (as shown in Fig. 1), and reported a substantial amount of cases where this angle was above $180^{\circ}$. These cases were named defects and they are only possible on account of particle friction. In a recent article, we revealed that the stability of arches against vibrations is related to these defects: the larger the defect, the lower the intensity of the vibration needed to break the arch [25]. In line with this result, Pournin et al. [26] showed the crucial role of friction for the clogging propensity of a granular material.

Apart from the applied interest of studying arches as promoters of clogging, it is desirable to clarify whether or not the properties of particles within these arches resemble the ones found in particles within the bulk of a granular sample. In this work, we perform numerical simulations that are compared to and validated against previous experimental results. Subsequently, we exploit the potential of the numerical simulations to analyze the forces acting on the particles belonging to the clogging arches and compare them with the forces taking place among particles in the silo bulk.

\section{Model}

To simulate clogging arches like the ones obtained experimentally in $[24,25]$, we have performed a discrete element modeling (DEM) [27] of a system of monodisperse spheres with diameter $d=1 / 32 \mathrm{~m}$. The particles are confined within a quasi-2D rectangular container of rigid walls, which have the same mechanical properties as the particles. On the perpendicular direction $y$ (off-plane), the gap between the two walls is $1.2 \times d$, and on the horizontal direction $x$ the silo is $32 \times d$ wide (see Fig. 1). At the bottom of the silo, an aperture is created using two particles whose centers are a distance $4 \times d$ apart. Hence the outlet size $R$, measured in particles diameters, is $R=3$ as depicted in Fig. 1 .

The experimental scenario of spontaneous development of stable arches is captured by generating a random granular gas within the container, and letting the particles fall down under the action of gravity. The lateral and bottom boundaries are built of fixed particles with similar mechanical properties. The particles flow through the orifice, where periodic boundary conditions are applied. The eventual formation of an stable arch stops the flow. The system is let to relax until the mean kinetic energy of the grains is several orders of magnitude smaller than the potential energy, i.e. $\frac{E_{c}}{E_{p}}<10^{-4}$.

In the simulation, each particle $i(i=1, \ldots, N)$ has three translational degrees of freedom and the rotational movement is described by a quaternion formalism [28]. The interaction between particle $i$ and particle $j, \mathbf{F}_{i j}$, is decomposed as:

$\mathbf{F}_{i j}=\mathbf{F}_{i j}^{N}+\mathbf{F}_{i j}^{T}$

where $\mathbf{F}_{i j}^{N}$ is the component on the normal direction to the contact plane. In the same way, $\mathbf{F}_{i j}^{T}$ is the component acting on the tangential direction. In our approach, the elastic normal interaction $\mathbf{F}_{i j}^{N}$, is defined by a linear contact. Moreover, to introduce dissipation, a velocity dependent viscous damping is assumed. Hence, the total normal force reads as:

$\mathbf{F}_{i j}^{N}=-k^{N} \delta \cdot \hat{\mathbf{n}}-\gamma^{N} m_{e f f} \cdot \mathbf{v}_{r e l}^{N}$,

where $k^{N}$ is the spring constant on the normal direction, $m_{r}=m_{i} m_{j} /\left(m_{i}+m_{j}\right)=m / 2$ stands for the pair's reduced mass, $\gamma^{N}$ is the damping coefficient in the normal direction and $\mathbf{v}_{r e l}^{N}$ is the normal relative velocity between $i$ and $j$. Moreover, the tangential force $\mathbf{F}_{i j}^{T}$ also includes an elastic term and a tangential frictional term accounting for static friction between the grains. This takes into account the Coulomb friction constraint as:

$\mathbf{F}_{i j}^{T}=\min \left\{\left|-k^{T} \xi-\gamma^{T} m_{r} \mathbf{v}_{r e l}^{T}\right|, \mu\left|\mathbf{F}_{i j}^{N}\right|\right\}$,

where $\gamma^{T}$ is the damping coefficient in the tangential direction, $\mathbf{v}_{\text {rel }}^{T}$ is the tangential component of the relative contact velocity of the overlapping pair, $\mu$ is the friction coefficient of the particles, and $|\xi|$ accounts for the elongation of an 
imaginary spring with elastic constant $k^{T}$. As long as there is an overlap between the interacting particles, $\boldsymbol{\xi}$ increases as $d \boldsymbol{\xi} / d t=\mathbf{v}_{r e l}^{T}$ [27]. Moreover, the tangential spring is truncated and renormalized as necessary to be orthogonal to the normal vector [29].

The Newton's equations of motion are integrated using a Fincham's leap-frog algorithm (rotational) [30] and a Verlet Velocity algorithm (translational) [31]. The kinematic tangential relative displacement, $\boldsymbol{\xi}(t)$, is updated using an Euler's algorithm. The value of the normal elastic constant is $k^{N}=10^{7} \mathrm{~N} / \mathrm{m}$ and the density of the particles $\rho=7580 \mathrm{~kg} / \mathrm{m}^{3}$. The particles stiffness has been chosen guaranteeing that the particle overlapping is less than the $2 \%$ of the particle radius. The tangential elastic constant was $k_{t}=2 / 7 \mathrm{k}^{N}$ and dissipation parameters $\gamma^{N}=725 \mathrm{~s}^{-1}$ and $\gamma^{T}=72.5 \mathrm{~s}^{-1}$ were used. The gravity is set to $g=10 \mathrm{~m} / \mathrm{s}^{2}$ and the time step to $\Delta t=10^{-6} \mathrm{~s}$. In the simulations, we have kept constant the previous set of parameters modifying only the friction coefficient. To get good statistics, the results presented below correspond to at least eight hundred stable arches for each case.

\section{Spatial morphology of clogging arches}

Clogging arches are multi-particle structures found just above the orifice. To identify those arches we consider the same protocol used in previous studies [24,25,32]. The method was formulated with a goal of being consistent with previous definitions of arches [1-8] where it is stated that the beads that effectively belong to an arch are those that are mutually stabilized. In other words, particles belonging to an arch are those that would collapse if any other bead in the arch is removed.

The experimental procedure used to define the particles belonging to a clogging arch is based in the identification of the first line of beads in mutual contact that are just above the orifice. To this end, first, the silo base at the left and right of the orifice is identified. Departing from one side, the bead in contact with the base which is closer to the orifice is tagged. Beginning from it, the beads in contact with it are listed, and the angles to the centers of the beads are calculated; the first angle among them (starting from the downward direction) is selected, and the corresponding bead is labeled as the next one in the arch. The procedure is repeated until the bead is in contact with the base at the other side. Finally, the particles in contact with the bottom of the silo are not considered to be members of the bridge as they indeed are conforming the base of the arch (see Fig. 1). Some subtleties concerning the procedure are discussed in [24].

Once the clogging arches are identified, we have analyzed the number of beads $\eta$ belonging to each arch. In Fig. 2 we show the probability density function (PDF) found in experi-



Fig. 2 (Color online) Probability density function (PDF) of the number of beads $\eta$ in the clogging arches. Symbols are used to display numerical results obtained for $R=3$ with different friction coefficients as indicated in the legend. Solid (dashed) line represents experimental data, previously reported in [24] using steel particles and $R=3.03$ $(R=3.61)$

ments [24] using steel particles and outlet sizes $R=3.03$ and $R=3.61$. Clearly, the number of particles depends on the orifice size, because the bigger the outlet, the larger the number of particles needed to form a blocking arch. Along with those experimental data, we present results from simulations performed with an orifice size $R=3.00$ and different values of $\mu$. It seems that the friction coefficient does not have a significant effect, even though larger arches (with more particles) are formed as $\mu$ increases. The comparison of numerical and experimental results suggests the same qualitative behavior. Nevertheless, slightly larger arches are found using the numerical scheme. We attribute this to the fact that the shape of the orifice in the numerical simulations is different from the experimental one. Experimental arches were all obtained using an orifice with an inverted V-shape [24,25] which enables the formation of smaller arches than the orifice shape implemented numerically. Indeed, the size distribution of numerical arches seems equivalent to the one obtained experimentally for $R=3.61$. In any case, the trend displayed by both distributions is similar: there is a maximum around $\eta=$ $R+2$, and then the probability decays exponentially as it was previously reported both in bulk [3] and clogging arches [24].

The validity of the numerical method is also assessed by checking the distribution of angles of the beads in the arch, as defined by the segments between the center of mass of the particles (see Fig. 1). In all the cases, i.e. experiments using steel particles with $R=3.03$ and $R=3.61$, and simulations with $R=3$ and three different friction coefficients, it is inferred that the shape of the distribution does not strongly depend neither on the outlet size, nor on the friction coefficient, as shown in Fig. 3. The curves show an asymmetric distribution 




Fig. 3 (Color online) PDF for the angles $\phi$ associated to particles in the arch. In the inset, the same PDF is shown in semilogarithmic scale. Symbols are used to display numerical results obtained for $R=3$ using different friction coefficients as indicated in the legend. Solid (dashed) line represents experimental data using steel particles and $R=3.03$ $(R=3.61)$. The dotted vertical line indicates $\phi=180^{\circ}$, the angle above which a particle is considered a defect

with a maximum at around $150^{\circ}-160^{\circ}$ and a faster decay for big angles than for small ones. However, the decay rates of the distribution tails are slightly different.

Generally speaking, it can be stated that the higher the friction coefficient, the wider the distribution of angles. This effect is specially enhanced for high values of $\phi$. As could be expected, increasing $\mu$ leads to slower decays in the distribution (see inset of Fig. 3). The fact that lower values of $\phi$ arise when increasing $\mu$ can be explained by the inverse correlation among the angles corresponding to consecutive particles within the arch. It was observed that a large angle is usually between two small angles, and viceversa [24]. These outcomes suggest that high values of friction coefficient enable the formation of more complex stable structures. The same trend (proliferation of large angles) is observed when the outlet size is increased, because as $\eta$ increases, the more likely it is that a larger $\phi$ appears [25].

\section{Force heterogeneity in the silo}

In the previous section, we have seen that the numerical simulations are capable of generally capturing the geometrical features of arches as observed in experiments. The numerical scheme also allows us to obtain the contact forces acting on the particles in the silo after a clogging event. Our first goal is to provide a picture of the force magnitude at different regions of the silo. In agreement with recent findings $[33,34]$, we have found there are important inhomogeneities both on the horizontal and vertical directions. Hence, the magnitude of the normal force has been studied for particles
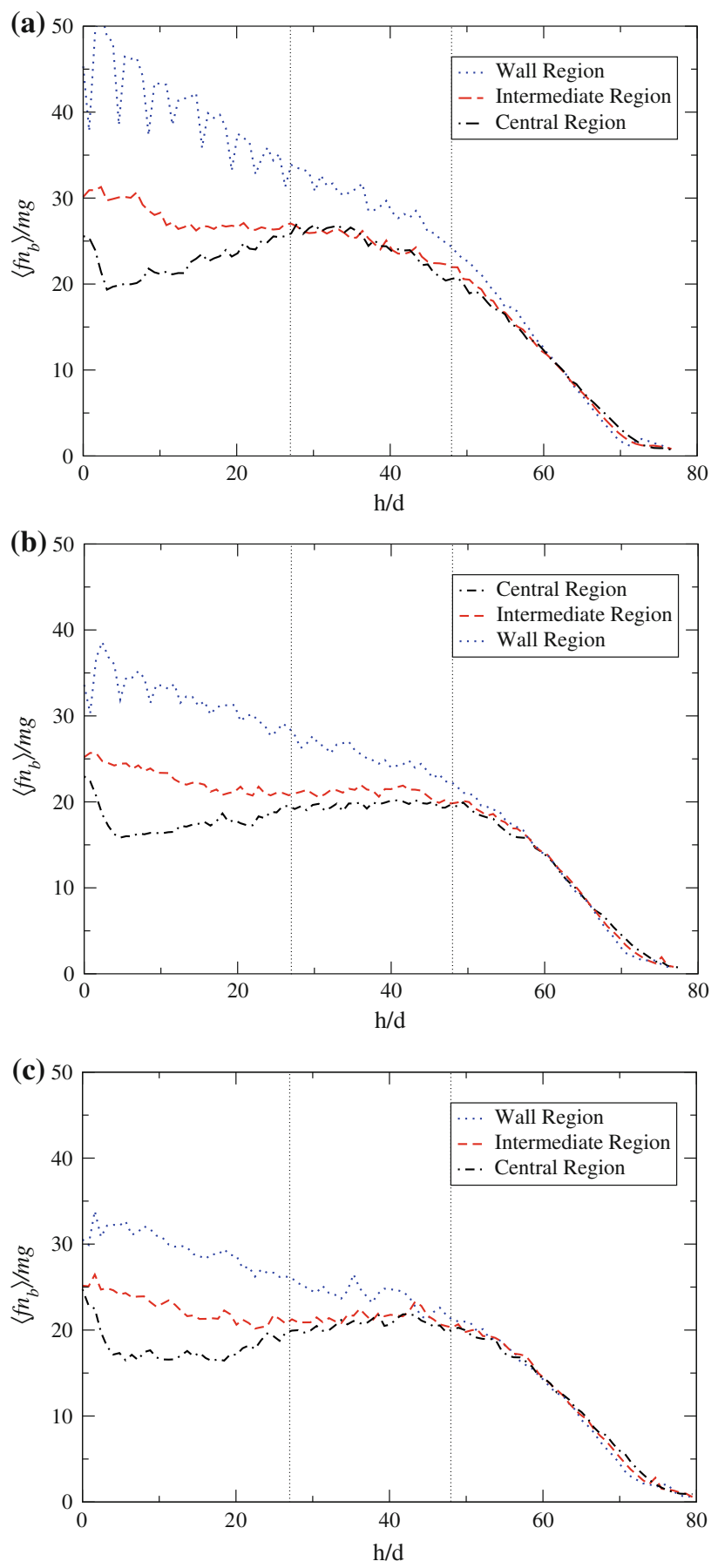

Fig. 4 (Color online) Profiles of the mean normal force along the height $(h)$ rescaled by the particle diameter $(d)$. Different profiles are shown for increasing values of friction: $\mathbf{a} \mu=0.2$, b $\mu=0.4$, and c $\mu=0.8$. In addition, results for different regions of the silo are shown in each graph (wall, intermediate, and centre, as indicated in Fig. 1). Vertical dotted lines indicate the heights that enclose the region $(27<h / d<48)$, which has been used to analyze the 'bulk' properties as explained in the text

located in three different regions of the silo: wall region (W), central region (C) and intermediate region (I) (the layout of these regions is indicated in Fig. 1). In Fig. 4 we present the 
profiles of the average normal forces vs. the height $h$. The trends obtained for different friction coefficients (plots (a), (b) and (c) in Fig. 4) are very similar. Starting from the surface $(h=80$ days $)$, the average normal force in the three regions $(\mathrm{C}, \mathrm{I}$ and $\mathrm{W}$, represented in different colors in each plot of Fig. 4) displays the same values, increasing linearly as $h$ decreases. At a height $h \approx 50$ days the force profiles begin to behave slightly different in these three regions. Note that this location (where the depth is similar to the silo diameter) coincides with the depth at which a pressure saturation is expected due to the Janssen effect. Accordingly, for $h<50$ days the average normal force in the central region of the silo decreases with $h$. This can be a reminiscence of the 'fluid state', in which it has been observed that the pressure is reduced as the orifice is approached [35].

On the contrary, near the walls $\langle f n\rangle$ increases as $h$ is reduced, although the slope of the profile is considerably smaller than the one obtained for higher positions. Finally, a halfway behavior is observed for the intermediate region I, where the force profile seems to saturate for $h<50$, with a slight increase of $\langle f n\rangle$ below $h \approx 20$ days.

The effect of modifying the particles' friction on the profiles of the average normal force (displayed in Fig. 4) can be summarized in two main issues: (1) low values of $\mu$ lead to higher values of normal forces in the wall region. (2) The effect of friction in the profiles of the central region is quite small. Those two effects lead to an enhancement of the differences among the central and lateral regions as $\mu$ is reduced. Another consequence of changing the friction coefficient of the particles is that the lower $\mu$ is, the closer to the surface the profiles begin to display differences.

As pointed out above, we have found important inhomogeneities in the silo. We will need a reference value for the next section of this paper, where we will compare the magnitude of normal and tangential forces in the arches and in the bulk of the silo. We have decided to take as a reference the mean normal force at the central and intermediate region for a height ranging from $h / d=27$ to $h / d=48$. This region is indicated by vertical dotted lines in Fig. 4 and it has a fairly uniform average normal force that, from now on, will be named $\left\langle f n_{b}\right\rangle$, i.e mean normal force at the bulk. The values of $\left\langle f n_{b}\right\rangle$ obtained for different friction coefficients are presented in Table 1.

Table 1 Mean values of the normal forces in terms of $m g$, measured in the arches $\left(\left\langle f n_{a}\right\rangle\right)$ and in the bulk region as defined in the text $\left(\left\langle f n_{b}\right\rangle\right)$

\begin{tabular}{llll}
\hline$\mu$ & 0.2 & 0.4 & 0.8 \\
$\left\langle f n_{b}\right\rangle$ & $24 \pm 2$ & $20 \pm 1$ & $21 \pm 2$ \\
$\left\langle f n_{a}\right\rangle$ & $44 \pm 3$ & $35 \pm 3$ & $32 \pm 2$ \\
\hline
\end{tabular}

The uncertainty of the mean values is expressed in terms of the $95 \%$ confidence interval of an exponential distribution

\section{Forces acting on particles in clogging arches and within the bulk}

Let us begin this section by remarking an interesting behavior revealed in all the profiles of the central region: when the distance to the bottom of the silo is smaller than 5 particle diameters, an augment of the normal force is observed as $h$ is reduced (i.e. as the outlet is approached). It is plausible that this increase of the forces near the orifice could be due to the presence of clogging arches which are, of course, only present in the central region of the silo. We have actually calculated the mean normal force $\left\langle f n_{a}\right\rangle$ within the clogging arches (see results in Table 1) and it is considerably higher than the average normal force in the central region for low values of $h$.

Taken at face value, these high values of mean normal forces found in the clogging arches seem to challenge the prediction of the simplified approximation introduced in [25]. In that work, it was suggested that the normal forces acting on the particles where the arch breaks ('the weakest link') are smaller than $10 \mathrm{mg}$. The discrepancy could be due to several causes. First, the average normal force calculated here takes into account all the particles belonging to the arch, while in the approximation of [25] only the particle promoting the arch collapse was considered. Second, it should be remarked that the numerical scheme only takes into account tangential forces due to static frictional effects, but no rolling friction or torsional deformation have been implemented -aspects which could be relevant to describe the stability of arches. And third, in order to simplify the analytic description, an approximation was introduced in [25] so that only horizontal defects were considered, where normal forces could be smaller than in defects with other orientations. To shed light on the origin of this discrepancy, it seems natural to examine if the normal forces in the arch have any dependence on their associated angle.

Figure 5 illustrates the average normal and tangential forces acting on particles that conform the arches. Specifically, we show the dependence on the angle $\phi$ associated to each particle for three values of friction coefficient. Interestingly, it seems that the trends displayed for both normal and tangential curves are similar for all the cases. Except for very small angles $\left(\phi<90^{\circ}\right)$ normal forces increase with $\phi$ for small values of $\phi$, reaching a maximum at around $180^{\circ}$, and then decrease as $\phi$ exceeds this value. This lowering of the normal forces at the defects may be the key to an accordance with the results presented in [25]. In addition, we believe that this phenomenon may be the cause of the behavior of normal forces observed in the interval $90^{\circ}<\phi<130^{\circ}$. As noted, in [24] it was found that consecutive particles belonging to the arch display an inverse correlation in their associated angles. Then, it seems reasonable that if particles with large angles have small normal forces, the same holds for particles with 


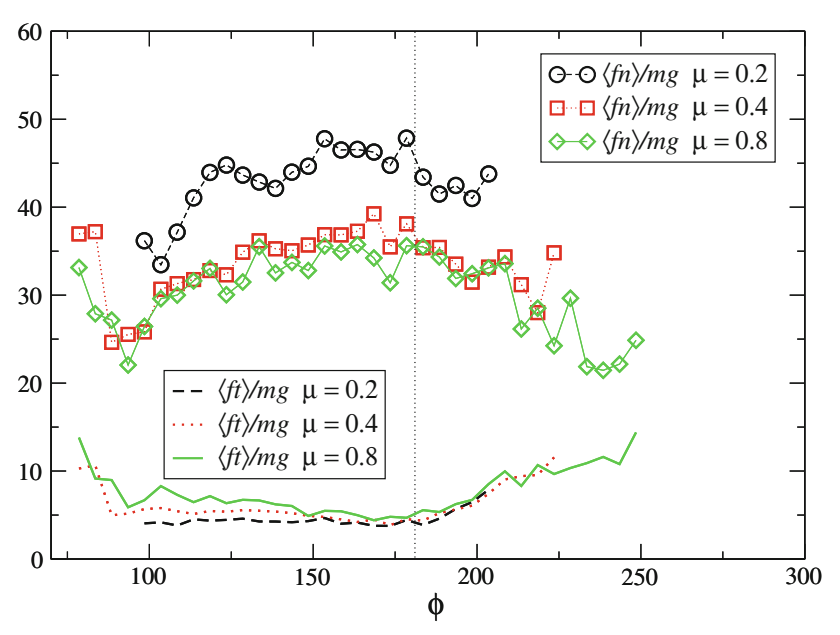

Fig. 5 Mean value of the normal and tangential force acting on all the particles in the arches, as a function of $\phi$. Each set of symbols corresponds to a different value of friction coefficient $\mu$, as indicated in the legend

small angles. From this, it follows that regular arches without defects are those that can withstand higher normal forces.

The behavior obtained for the tangential forces in Fig. 5 is the opposite than for normal forces. Indeed, tangential forces are almost zero for intermediate values of $\phi$, increasing notably as $\phi$ grows above $180^{\circ}$. This is an expected result as tangential forces are necessary to stabilize angles above $180^{\circ}$ and hence, the higher the angle is, the stronger the tangential force. Let us now describe the effect of the interparticle friction in the force dependence on $\phi$. Firstly, the normal forces in the arch are considerably higher for $\mu=0.2$ than for the cases of higher friction, where the outcomes are very similar. Another expected trend revealed by Fig. 5 is that the higher the friction coefficient, the higher the tangential forces and the angles that can be reached within the arches. Consequently, the decrease of normal forces for $\phi>180^{\circ}$ is sharper when friction increases.

Once we have found that the forces in the arch seem to display special properties, we analyze the distribution of forces within the arch and compare it with the distribution of forces in the bulk. Figure 6 displays the distribution of normal forces in the bulk and in the arch. In both cases, to allow straight comparison, we have rescaled the normal forces by the average normal force of the particles in the bulk $\left\langle f n_{b}\right\rangle$. Interestingly, the distributions are quite different. For the bulk, we obtain the typical monotonous exponential decay that is often observed in granular materials. On the contrary, the force distribution in the arch exhibits a narrower distribution with a maximum that has been typically attributed to the development of yield stress and force chains [36-39]. Indeed, it is expected that in arches, the values of forces lie around the average as the forces acting on a particle must be compensated. In addition, from Fig. 6 it is evidenced that the distribution of forces around the average has not a significant dependence on the friction coefficient.

The tangential force distributions (Fig. 7) reveal a behavior similar to the normal ones. For the particles in the bulk, a monotonous exponential decay is observed. For the particles belonging to the arches, we obtain a narrower distribution with an apparent maximum which is, however, less pronounced that the one encountered in the arch distribution of normal forces. The differences among the tails evidenced for the exponential distributions using distinct friction coefficients can be attributed to the rescaling factors which, as explained above, are the average normal forces in the bulk for each case.

Finally, the role of friction at the bulk and in the clogging arches is examined with the distribution of the mobilized friction $\frac{f t}{\mu f n}$ [39] (see Fig. 8a, b). Those distributions indicate how far away the contacts are from the Coulomb threshold criterion. In general, the outcomes are very similar for both the bulk and the arches, revealing that the higher $\mu$ is, the harder to reach the threshold criterion $\frac{f t}{\mu f n}=1$. Moreover, the data show that most of the contacts are much below the Coulomb failure condition. Those results suggest that even when very disordered arch morphologies are obtained, the majority of the contacts are far away from the Coulomb threshold criterion. In addition, it is reasonable to think that the few particles that are closer to the condition $\frac{f t}{\mu f n}=1$ are those with an associated angle $\phi>180^{\circ}$, as observed in Fig. 5. This correlates with our weakest-link argument for breaking arches [25].

\section{Conclusions}

In this work, we have analyzed the forces developed among the particles belonging to the arches that clog the exit of a silo. The first important result is that the magnitude of the average normal force within particles in an arch is considerably larger than the normal forces in the particles around it. Furthermore, we observe a strong dependence of the forces in the arch on the angle associated to each particle (the angle subtended from the particle center to the contact points with its two neighbors). For defects, the larger the angle, the higher the tangential force and the lower the normal one. The first result was somehow expected and the second one seems to be in agreement with the low values of normal force obtained from the simplified argument recently put forward to explain the resistance of arches against vibrations [25]. Finally, we explore the distribution of normal and tangential forces within arches and compare it with the forces acting on particles in the bulk. For the case of clogging arches, both distributions reveal a well defined maximum indicating their chain-like properties. However, for the case of the bulk the distribution is wider, revealing the typical monotonous expo- 


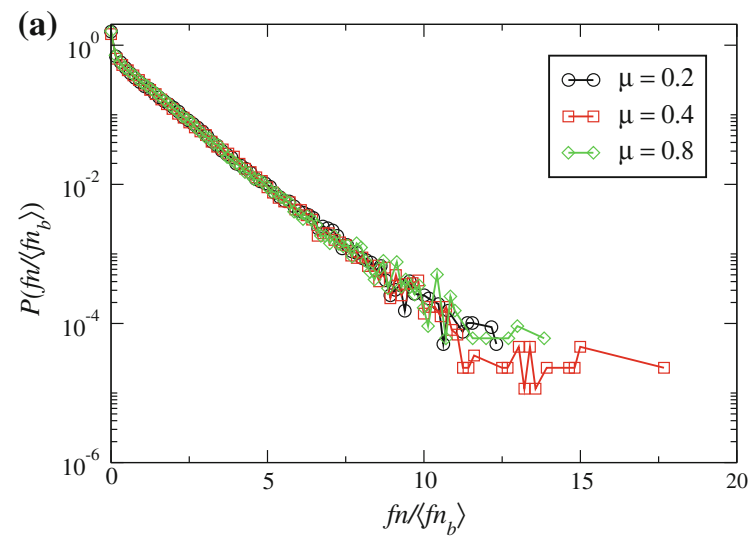

Fig. 6 (Color online) a PDF of the normal forces of the particles in the bulk rescaled by the average normal force in the bulk. b PDF of normal forces of the particles in the arch rescaled by the average normal

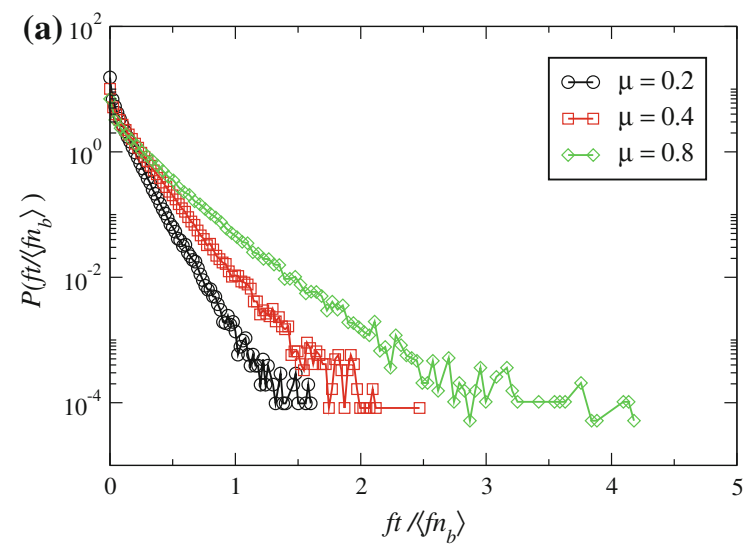

Fig. 7 (Color online) a PDF of tangential forces of the particles in the bulk rescaled by the average normal force in the bulk. b PDF of tangential forces of the particles in the arch rescaled by the average normal

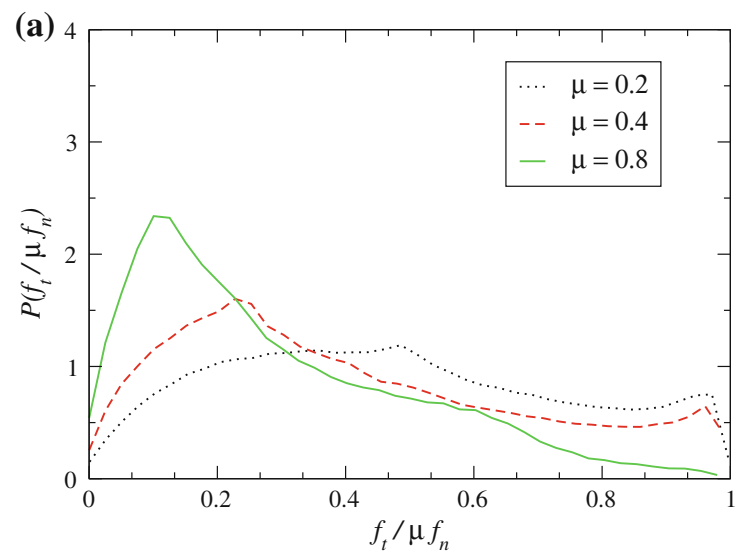

Fig. 8 (Color online) a PDF of the mobilized friction $\frac{f t}{\mu f n}$ of forces acting on particles at the bulk. b PDF the mobilized friction $f n / f t$ of forces acting on particles at the arches.



force in the bulk. In both graphs, different symbols correspond to the simulations performed varying the friction coefficient $(\mu)$ as indicated in legends

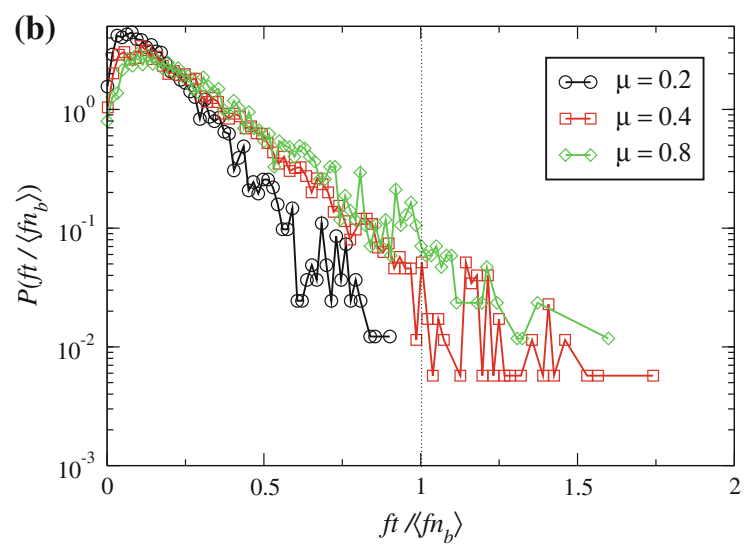

force in the bulk. In both graphs, different symbols correspond to the simulations performed varying the friction coefficient $(\mu)$ as indicated in legends

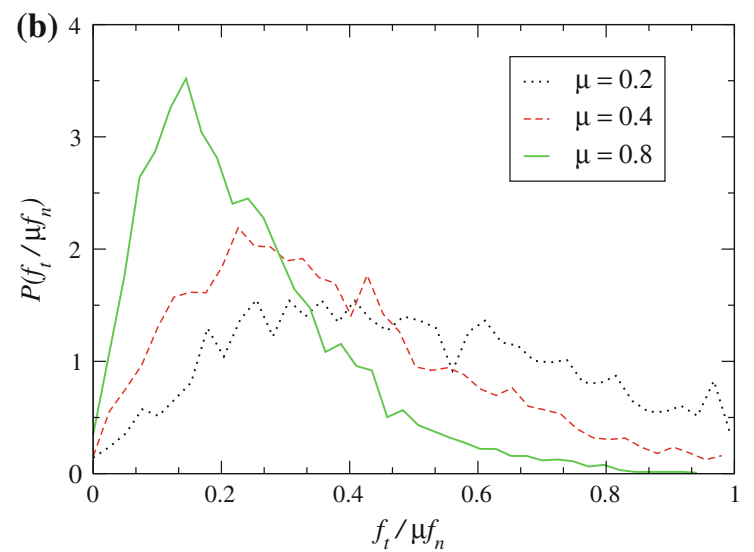

In both graphs, different symbols correspond to the simulations performed varying the friction coefficient $(\mu)$ as indicated in legends 
nential decay of granular materials that have not developed yield stress. These results manifest the unique features of the arches that block the orifice, suggesting the local character of the clogging process.

Acknowledgments We thank D. Maza and L. A. Pugnaloni for discussions, S. M. Rubio-Largo for help with Fig.1, and L. F. Urrea for technical help. This work has been financially supported by Project FIS2011-26675 (Spanish Government), and PIUNA (Universidad de Navarra). C. L. thanks Asociación de Amigos de la Universidad de Navarra for a scholarship.

\section{References}

1. Pugnaloni, L.A., Barker, G.C., Mehta, A.: Multi-particle structures in non-sequentially reorganized hard sphere deposits. Adv. Complex Syst. 4, 289 (2001)

2. Pugnaloni, L.A., Barker, G.C.: Structure and distribution of arches in shaken hard sphere deposits. Physica A 337, 428 (2004)

3. Mehta, A., Barker, G.C., Luck, J.M.: Cooperativity in sandpiles: statistics of bridge geometries. J. Stat. Mech. Theory Exp. 10, P10014 (2004)

4. Cao, Y.X., Chakrabortty, B., Barker, G.C., Mehta, A., Wang, Y.J.: Bridges in three-dimensional granular packings: experiments and simulations. EPL 102, 24004 (2013)

5. Arévalo, R., Maza, D., Pugnaloni, L.A.: Identification of arches in 2D granular packing. Phys. Rev. E 74, 021303 (2006)

6. Carlevaro, C.M., Pugnaloni, L.A.: Arches and contact forces in a granular pile. Eur. Phys. J. E 35, 1 (2012)

7. Jenkins, M.C., Haw, M.D., Barker, G.C., Poon, W.C.K., Egelhaaf, S.U.: Does gravity cause load-bearing bridges in colloidal and granular systems? Phys. Rev. Lett. 107, 038302 (2011)

8. Jenkins, M.C., Haw, M.D., Barker, G.C., Poon, W.C.K., Egelhaafab, S.U.: Finding bridges in packings of colloidal spheres. Soft Matter 7, 684 (2011)

9. Helbing, D., Buzna, L., Johansson, A., Werner, T.: Self-organized pedestrian crowd dynamics: experiments, simulations, and design solutions. Transp. Sci. 39, 1 (2005)

10. Parisi, D.R., Dorso, C.O.: Microscopic dynamics of pedestrian evacuation. Physica A 354, 606 (2005)

11. Guariguata, A., Pascall, M.A., Gilmer, M.W., Sum, A.K., Dendy Sloan, E., Koh, C.A., Wu, D.T.: Jamming of particles in a twodimensional fluid-driven flow. Phys. Rev. E 86, 061311 (2012)

12. Lafond, P.G., Gilmer, M.W., Koh, C.A., Dendy Sloan, E., Wu, D.T., Sum, A.K.: Orifice jamming of fluid-driven granular flow. Phys. Rev. E 87, 042204 (2013)

13. To, K., Lai, P.-Y., Pak, H.K.: Jamming of granular flow in a twodimensional Hopper. Phys. Rev. Lett. 86, 71 (2001)

14. To, K., Lai, P.-Y.: Jamming pattern in a two-dimensional hopper. Phys. Rev. E 66, 011308 (2002)

15. Zuriguel, I., Pugnaloni, L.A., Garcimartín, A., Maza, D.: Jamming during the discharge of grains from a silo described as a percolating transition. Phys. Rev. E 68, 030301 (2003)

16. Zuriguel, I., Garcimartín, A., Maza, D., Pugnaloni, L.A., Pastor, J.M.: Jamming during the discharge of granular matter from a silo. Phys. Rev. E 71, 051303 (2005)
17. Sheldon, H.G., Durian, D.J.: Granular discharge and clogging for tilted hoppers. Granul. Matter 12, 579 (2010)

18. Thomas, C.C., Durian, D.J.: Geometry dependence of the clogging transition in tilted hoppers. Phys. Rev. E 87, 052201 (2013)

19. Zuriguel, I., Janda, A., Garcimartín, A., Lozano, C., Arvalo, R., Maza, D.: Silo clogging reduction by the presence of an obstacle. Phys. Rev. Lett. 107, 278001 (2011)

20. Saraf, S., Franklin, S.V.: Power-law flow statistics in anisometric (wedge) hoppers. Phys. Rev. E 83, 030301(R) (2011)

21. Magalhaes, C.F.M., Moreira, J.G., Atman, A.P.F.: Segregation in arch formation. Eur. Phys. J. E 35, 38 (2012)

22. Janda, A., Zuriguel, I., Garcimartín, A., Pugnaloni, L.A., Maza, D.: Jamming and critical outlet size in the discharge of a twodimensional silo. Europhys. Lett. 84, 44002 (2008)

23. Longjas, A., Monterola, C., Saloma, C.: Force analysis of jamming with disks of different sizes in a two-dimensional hopper. J. Stat. Mech. 05, 05006 (2009)

24. Garcimartín, A., Zuriguel, I., Pugnaloni, L.A., Janda, A.: Shape of jamming arches in two-dimensional deposits of granular materials. Phys. Rev. E 82, 031306 (2010)

25. Lozano, C., Lumay, G., Zuriguel, I., Hidalgo, R.C., Garcimartín, A.: Breaking arches with vibrations: the role of defects. Phys. Rev. Lett. 109, 068001 (2012)

26. Pournin, L., Ramaioli, M., Folly, P., Liebling, ThM: About the influence of friction and polydispersity on the jamming behavior of bead assemblies. Eur. Phys. J. E 23, 229 (2007)

27. Pöschel, T., Schwager, T.: Computational Granular Dynamics. Springer, Berlin (2005)

28. Wang, Y.C., Abe, S., Latham, S., Mora, P.: Implementation of particle-scale rotation in the 3-d lattice solid model. Pure Appl. Geophys. 163, 1769 (2006)

29. Weinhart, T., Thornton, A.R., Luding, S., Bokhove, O.: Closure relations for shallow granular flows from particle simulations. Granul. Matter 14, 531 (2012)

30. Fincham, D.: Leapfrog rotational algorithms. Mol. Simul. 8, 165 (1992)

31. Verlet, L.: Computer "experiments" on classical fluids. II. Equilibrium correlation functions. Phys. Rev. 165, 201 (1968)

32. Lozano, C., Janda, A., Garcimartín, A., Maza, D., Zuriguel, I.: Flow and clogging in a silo with an obstacle above the orifice. Phys. Rev. E 86, 031306 (2012)

33. Staron, L., Lagrée, P.-Y., Popinet, S.: The granular silo as a continuum plastic flow: the hour-glass vs the clepsydra. Phys. Fluids 24, 103301 (2012)

34. Perge, C., Aguirre, M.A., Gago, P.A., Pugnaloni, L.A., Le Tourneau, D., Géminard, J.C.: Evolution of pressure profiles during the discharge of a silo. Phys. Rev. E 85, 021303 (2012)

35. Rubio-Largo, S.M., Janda A., Maza D., Hidalgo R.C.: In preparation

36. Mueth, D.M., Jaeger, H.M., Nagel, S.R.: Force distribution in a granular medium. Phys. Rev. E 57, 3164 (1998)

37. O’Hern, C.S., Langer, S.A., Liu, A.J., Nagel, S.R.: Force distributions near jamming and glass transitions. Phys. Rev. Lett. 86, 111 (2001)

38. Corwin, E.I., Jaeger, H.M., Nagel, S.R.: Structural signature of jamming in granular media. Nature 435, 1075 (2005)

39. Majmudar, T.S., Sperl, M., Luding, S., Behringer, R.P.: Jamming transition in granular systems. Phys. Rev. Lett. 98, 58001 (2007) 